\title{
Performance Analysis of Cloud-based Health Care Data Privacy System Using Hybrid Techniques
}

\author{
Sapna Kumari C. ${ }^{1}$, Asha C. N. ${ }^{2}$, Rajashekhar U. ${ }^{3}$, K. Viswanath ${ }^{4}$ \\ ${ }^{1}$ Department of ECE, Nitte Meenakshi Institute of Technology, Bengaluru, Karnataka, India. sapna.kumari@nmit.ac.in \\ ${ }^{2}$ Department of ECE, Acharya Institute of Technology, Bengaluru, India, ashacn@acharya.ac.in \\ ${ }^{3}$ Department of E \& C Engg., Government Engineering College, uraju54@gmail.com \\ Senior Software Engineer, Ajitek Technology Solutions Pvt. Ltd, Hyd, viswa4vlsi@gmail.com
}

Received: June 12, 2021. Revised: November 20, 2021. Accepted: December 10, 2021. Published: January 3, 2022.

\begin{abstract}
At present, due to the various hacking approaches, the protection for any data transmitted through any channel or mode is one of the important issues. Nowadays, providing data security is satisfactory, developments are extended for obtaining data among the transceivers. Security level depends on the size of a symmetric key which is employed for encryption and decryption using various cryptography systems management and in modern approaches like block and RF codes including AES use a larger size of key simultaneously and there exists security problems due to hacking approaches. To illustrate the protection level and hacking problems, a new ECC is presented as well as by employing scalar duplication, the synchronous key is generated and consists of point doubling and point addition. The created focuses are encrypted before transmission by using ECC-Elgamal-Holomorphic (ECCEH) and transferred through a distant channel and encipher data is failed at the receiver using ECCEH which includes the reverse process. The unique standards of cryptography context have been generated by MATLAB; the defined framework has endeavored to the extent that speed, delay as well as control, and
\end{abstract}

\section{INTRODUCTION}

This era has observed an astronomical development in communications over wired and wireless networks. Every day the World Wide Web deals with thousands of transactions. Some of these communications have critical data that must be private, transactions are genuine, and users authentic. A strong-featured security structure is employed to execute these prerequisites. The science which deals in providing secure communications is termed Cryptology. The main objective of cryptology is to design techniques that allow only endorsed access to information. All malicious attempts to access information are avoided. The cryptographic key recognizes authorized to access. The hidden information is accessed by the user who is having a precise key, whereas other users will not have access to the information. Cryptology comprises cryptography and cryptanalysis. The earlier involves analysis and application of various techniques through which information may be inappropriate to all except the intended receiver. In contrast, the many others are accepted in MATLAB 2017a. The user of the sender, the original information is transformed into integer value by employing Holomorphic and encodes it by utilizing the Elgamal ECC algorithm which employs point doubling and point addition. The encoded information is uploaded into the cloud for storage, here www.thingspeak.com is utilized for storage. When the user presents at the receiver request the cloud to access from it, initially the cloud server authenticates the access control strategies of the requester, and then access is provided by the cloud server. If the user authenticates the strategies, then encoded data can download and the original data is decoded by synchronous key employing ECCElgamal algorithm. Using original and decrypted data, various performance factors are calculated in terms of execution time, packet delivery ratio, throughput, latency and compare these results with conventional methods and found to be $12 \%, 31 \%, 24 \%$, and $8 \%$ progress concerned with packet delivery ratio, latency, outturn and execution time.

Keywords: ECC, Elgamal, AES, Holomorphic, Cloud computing, Cryptography.

science which deals with breaking cryptosystems and recovering secret information is referred to as cryptanalysis. Symmetric key and asymmetric key algorithms are the two types of cryptographic algorithms. For both cipher-texting and deciphertexting, the data single key is employed in Symmetric key cryptographic algorithms. These methods are widely used. These are generally chosen because of their high speed and simplicity. However, they can be employed only when the two communicating parties have agreed on the secret key. Since it is not always easy for users to exchange keys this could become a major drawback in practical cases. Two keys are involved in asymmetric key cryptographic algorithms. A secret key and an open key. The secret key is kept private whereas the public key is known to everyone. The encryption is carried out with the help of the open key; by using the corresponding private key the cipher-texted message can be decrypted. The security of these algorithms can be determined based on the rigidity of deriving the secret key from an open key. However, slow and highly complicated, asymmetric key cryptography has huge 
benefits. The main benefit is that the basic primitives employed are based on well-known complications such as integer factorization and discrete logarithm issues. These difficulties have been examined widely and after years of research also their stability has not been denied. This is different from symmetric-key cryptography where the potency of the algorithm depends on combinatorial techniques. The security of such algorithms is not substantiated and does not depend on complications in literature. RSA is one of the widely used asymmetric key crypto algorithms [4].

Recently asymmetric crypto algorithms that are based on elliptic curves have become popular due to lower key sizes and achieves a higher level of security. Various security benchmarks have been developed which employ elliptic curves for the fundamental security algorithm. There are several approaches to cryptanalysis novel cryptographic algorithms. Typical Cryptanalysis methods make use of algorithm drawbacks. Due to the large data requirement, they cannot be practically achieved. Furthermore, large computation time is required by many of the approaches that make them highly expensive. On the other hand, the attacks on modern cryptographic algorithms experience serious threats depending on the information collected from side channels. These attacks detection proposed by $\mathrm{P}$. Scholl and C. Gentry [5-6] focuses on the execution rather than the algorithm. Sources of side-channel consist of power consumption of the device, timing, audibility, and radiation features; thus, an attacker examines one or more side channels of a device carrying out the encryption (or decryption) and can collect information about the secret key. Enhanced cryptographic performances are more exposed to side-channel attacks, thus high performing cryptographic hardware must take into account this class of attacks during execution.

Since the development is rapid, attempts are made to prevent the hacking of data by the study of researchers and industries. To exchange information via communication media securely, the best technique is ECC cryptography which is more secure than all other cryptography techniques discussed by H. Jing-Li [78]. The ECC generates varied keys sizes and for the given information, encoding and decipher-texting are performed. ECC employs private and public-key cryptography. Simultaneously, the Conventional key cryptography structure provides more security, accordingly, one such cryptography strategy is ECC. The basic advantage of ECC over RSA is that based on the applications and requirements ECC supports smaller keys to larger key sizes and as a result managing multifaceted quality will be reduced in Y. GovindaRamaiah.et. al [9]. In ECC point addition, point multiplication and scalar additions are basic operations to determine the focal point on a curve. This point operation facilitates decryption and encipher-texting functions. Elgamal approach is considered as a part of ECC operation and is one among such point operations similarly enhanced for statically synchronized and it operates in the same way as one to $\mathrm{N}$ mapping method explained in Arunkumar. et.al [10]. During mapping approach, the $\mathrm{x}-\mathrm{y}$ coordinator is mapped with the alphanumeric characters, numbers gradually and is extremely difficult for an intruder to identify the number or character which are mapped, hereafter the mapped approach is used in this context and is referred as grid mapping approach and assures security for the data and avoids encrypted information. Exchanging the information among the transceiver is the major drawback of the crypto technique which is confined using the ECC Diffie-Hellman-Merkle key exchange by carrying out a scalar addition to generate the focal points such as P, 2P, 3P, 4P up to 563P. Generated point $\mathrm{P}=\mathrm{f}(\mathrm{x}, \mathrm{y})$ obtains 563 focal points, point addition, point multiplication is used to reduce complex arithmetic operation such as addition and division; consequently, the casualness of framework is reduced and speed enhances by O.Srinivas Rao. et.al [11]. Reed Solomon codes and Elgamal strategy are employed by sender message to accelerate the process with $200 \mathrm{Mbits} / \mathrm{sec}$ transmission speed proposed by Riaz Naseer. et.al [12]. The message sent is stored in $\left(\mathrm{M}+\mathrm{M} \_\mathrm{s}^{*}\left(\mathrm{M} \_\mathrm{R}^{*} \mathrm{G}\right)\right)$, the message is represented by $\mathrm{M}$, the sender message is denoted as M_s, receiver message by M_R, generator point is given by $G$ and (M_s*G, M+M_S*(M_R*G)) encrypts the message and error variations in memory is presented in Juan Antonio Maestro [13]. The sender key KR and $\mathrm{K} \_\mathrm{R} *\left(\mathrm{M} \_\mathrm{s}^{*} \mathrm{G}\right)$ registers, with the help of specific key $M$ the information is decoded by the receiver by employing $\mathrm{M}+\mathrm{M} \_\mathrm{s}^{*}\left(\mathrm{M} \_\mathrm{R} * \mathrm{G}\right)-\mathrm{K} \_\mathrm{R} *\left(\mathrm{M} \_\mathrm{s}^{*} \mathrm{G}\right)$ in Gustavo Neuberger [14] and it is more consistent and reduces the additional bits in memory devices. To implement SoC and Network operation Center excess operations performed are reduced and these frameworks are straightforward and part of the codes is exhibited in SanghyeonBaeg [15]. Several bits of error modification, accurate fault resistance devices such as (CAMs) Content Addressable Memories, and accurate error correction used for memories such as SRAM and other memories devices are included in [16] by Sandeep M D.et.al. Data exchanging devices such as $\mathrm{NoC}$ and $\mathrm{SoC}$ structures require error modification techniques to decrypt the information at the receiver and information exchange is effectively shown in Costas Argyrides. et.al and Bertozzi, D., et al [17-18]. Regarding points representation for the characters, ElGamal is better than Menezes ECC systems and ElGamal, schemes consume less power and it operates at very high speed in ASIC, FPGA design and the fields considered over the curve are 
$F_{p} . F_{p}$ proposed by F.Amounas.et.al and Do-Hyeon Choi.et.al [20]. Later, the concept of ECC has been developed by the researchers; the elliptical curve plays a significant role in cryptography and employs number theory and security for the systems proposed by Prashant Sharma. et.al [19]. ECC technique is utilized in integer factorization numbers and is extremely useful to solve Fermat's theorem suggested in [21] discussed by Swadeep Singh. et.al.

In the year 1985 Koblitz and P. K. Shau developed ECC by P. K. Shau.et.al[22] and it is employed to generate a secret key which is practically available and provides a high level of security, the curve with respect to point $\mathrm{P}$ is denoted as $\mathrm{p}\left(\mathrm{x}_{[\mathrm{i}}, \mathrm{y}_{[\mathrm{j}]}\right)$ for all values of $\mathrm{P}$ and must satisfy the equation at infinity point which is given below and proposed by Hankerson. et.al and M. Kurt. et.al [24,23].

$$
\mathrm{y}_{\left[{ }^{2}\right]}+\mathrm{c}_{1} \mathrm{x}_{[\mathrm{i}]} \mathrm{y}_{[\mathrm{j}]}+\mathrm{c}_{3} \mathrm{y}_{[\mathrm{j}]}=\mathrm{x}^{3}{ }_{[\mathrm{i}]}+\mathrm{c}_{2} \mathrm{x}^{2}{ }_{[\mathrm{i}]}+\mathrm{c}_{4} \mathrm{x}_{[\mathrm{i}]}+\mathrm{c}_{6}
$$

The curve with respect to point $P$ for elliptical curve $K$ is specified as $P(K)$, the points on $P$ for all values $\mathrm{P}$ for many fields are outlined. The ECC field is generally expressed as complex numbers that are real and rational associated with finite curve field, to provide high security both fields employ prime number and complications in the ECC systems relies on the decoding capacity of the curve which is typically referred as DLP Sponsor LAN/MAN Standards Committee. ElGamal cryptography is one of the well-known algorithms in ECC which provides high security for both encryption and decryption is conferred in Fatima. et.al [25]. Diffie and Hellman presented cryptography by M. Kurt. et.al [23] in 1976 to substitute the key as an open key, in later stage, RSA has been designed for less key size discussed by $\mathrm{K}$ Subba Reddy. et.al [26]. IEEE 802.16 protocol is mostly used to transmit the information wirelessly for WiMAX protocol which is a part of the control and physical layer in OSI model and utilized in point-topoint communication which employs mesh type topology. Excluding physical and control layers, there are other layers that provide data security and authorize, verification of data is conferred. To encode the data, instead of using the control layer, a physical layer is used for the modulation of carrier signals and frequencies. MAC is a fundamental sub module for security and it functions as convergence and monitoring information transfer from sender to the recipient by M Purusotham Reddy. et.al [27]. In the ECC model, the novel approaches presented for data encipher-texting are Menezes Vanstone. This approach fundamentally divides the main unit into smaller blocks similar to the pipeline process and comprises one character in the format of hexadecimal. Each hexadecimal value possesses two digits to represent the data as a point by $\mathrm{T}$. Wollinger. et.al [28]. In a communication system, the existing period has observed a progressive development. In limited-resource environments, applications like mobile communication, PDAs, online banking, smartcards, and so on mainly focus on security. Due to small key sizes, elliptic curve cryptography (ECC) is considered as one of the ideal cryptographic tools and the security is comparable with other typical public algorithms. However, hardware acceleration of cryptographic algorithms is required to sustain the increasing demand for speed in contemporary applications. For instance, the techniques must be prone to side-channel intrusions. Adaptable hardware models for elliptic curve cryptography over binary Galois fields are explored in this research work. The competency is greatly influenced by fundamental arithmetic primitives. The thesis thus deals with the design of FPGA for two significant field primitives' viz. multiplication and inversion. Similar to software programs FPGAs are also considered as reconfigurable hardware Platforms that are low in cost and flexible. On the other hand, due to large functionality, large routing delay, and limited resources designing on FPGA platforms has become a significant task. A lookup table is the smallest programmable unit in an FPGA. The exploitation of LUTs on the FPGA is increased by using the arithmetic algorithms proposed in this thesis. A modern finite field multiplier based on the recursive Karatsuba algorithm is intended in Do-Hyeon Choi. et.al [29]. The designed multiplier is associated with two variants of Karatsuba, viz. the typical and the simple Karatsuba multipliers. The typical Karatsuba multiplier has a significant gate count except for small-sized multiplications as the multiplier employs LUT resources proficiently. The simple Karatsuba is efficient and employed for large-sized multiplications, as a smaller number of gates are required. The designed hybrid multiplier performs the initial recursion through the simple algorithm while final small-sized multiplications are realized by the general algorithm proposed by $\mathrm{M}$. IndraSena Reddy. et.al [30]. The multiplier so achieved has the best area and time outputs in contrast to reported literature. The Itoh-Tsujii multiplicative inverse algorithm is based on Fermat's little theorem and $m$ -1 squaring's and $\mathrm{O}(\log 2(\mathrm{~m}))$ multiplications are required. Quad-Itoh Tsujii is a proposed inverse algorithm, is based on an FPGA that employs quad circuits and is more efficient than making use of squarer due to an enhanced LUT exploitation. $(\mathrm{m}-1) / 2$ quad circuits are required by Quad-Itoh Tsujii that have the best process time when compared to any inverse algorithm described. The designed primitives are structured as an elliptic curve cryptoprocessor (ECCP) that provides the best timings and area time output in contrast to reported works. We conclude that, if the basic primitives are prudently designed then the functioning of an ECCP is considerably improved. Additionally, a side- 
channel attack based on simple timing and power analysis is exhibited on the ECCP. The structure of the ECCP is then customized to reduce such attacks.

\section{RELATED WORK}

A unique evaluation technique is proposed in this research work for suspecting criminals by utilizing social as well as criminal information that is obtained by the social network as well as police information systems to address the drawback of data security as well as data privacy. The social cloud server, as well as the public security cloud server, is facilitated to securely exchange social information about the suspected criminals as well as public info about users. A privacy-preserving information retrieval technique that is based on oblivious transfer is introduced in this work to authenticate users' organizations that request social data of the suspect while the social cloud server could not access any information during the data request. This work also introduces many building blocks that include a comparison of data encryption, securely classifying the data as well as the regression tree (CART) model. Therefore, we developed a privacy-preserving criminal suspects sensing methodology based on the above-mentioned building blocks. At last, a performance review that illustrates our strategy is presented and this strategy improves the interpretation of criminal suspects and thereby minimizes the security breaches as well as expenses [1]. The homomorphic algorithm is often employed in [1] to perform encryption as well as decryption process, which further involves more computational processes to obtain the encrypted data for transmitting.

The architecture makes use of a three-component differential-as well as-distortion privacy-preserving conceptual model to minimize the data quality loss that is caused by the misperception of location. To incorporate actual sensed information to the obfuscated place, first, we understand the function of data modification. The complexity in data adaptation is reduced by the linear program under the requirements of differential, distortion privacy, as well as evenly-distributed obfuscation. To minimize the number of computational resources, an approximated technique is presented in this work. Further, an uncertainty-aware inference technique to optimize the obfuscated data inference precision is presented. When compared to other conventional techniques, the proposed technique offers the same security level but minimizes the data quality loss by $42 \%$. The estimated technique imposes an extra $3 \%$ quality loss when compared to that of the optimal technique, but the approximated technique requires only $1 \%$ of the computation time [2]. In [2], the graph theory is applied to improving data privacy using uncertainty-aware inference which is a homogeneity function and it requires a lot of mathematical functions to obtain the final decrypted data. Therefore, the throughput and latency are the major problems in [2].

The evolution of medical applications in big data is very much essential for developing advanced health care and thereby expanding hospital management towards a digital, sophisticated, as well as scientific state. The development of regional smart health care service platforms provides more space for improvements in cloud computing technology, and the future outlook of smart health care cloud will be much excellent. On the other hand, using big data in the healthcare system seems to have its benefits as well as drawbacks. A health care system based on the fuzzy concept is presented in [3] which is developed to deal with the consequences of patient health records leakage from the resource, access behavior sensitivity, as well as past and present access information preceptive beginning with the summary of health care, management, as well as science, people-oriented, patient-centered, study on data security under the environment of big data health care. It is crucial to achieving the reliability as well as precision of risk management because of the unavailability of accurate data in real problemsolving processes. As a result, with the help of fuzzy rule technology as well as from the uncertainty of risk factors, the analysis is carried out in real-time applications and thereby enhancing the efficiency of risk assessment techniques [3]. In [3] proposed fuzzy-based encryption and decryption process which requires less computational operations but the security level is not up to level.

\section{Problem Statement}

Based on the survey from the latest articles published in IEEE transactions and Springer publications on data privacy and security levels, the health care big data and police information are major concerns for transmission through wireless or wired communications with help of encryption and decryption process. The maximum accuracy obtained from the existing work-related data privacy is $90 \%$ and it uses the homomorphic system to obtain the cipher output. The computational operations required for performing the cipher output for more privacy and security are not acceptable for present communication technology. The key generation is another challenging task for encryption and decryption process in the existing system, these keys can easily attacks by a third person in between sender and receiver, therefore the data privacy and security levels are needed to be improved for the current communication technologies. 


\section{METHODOLOGY OF PROPOSED RESEARCH WORK}

From the hasty growth of contemporary more important information technology, the medical information or secrete information of police or military information sharing from one to one becomes a challenging area in real-world communications. Mainly health care industry information and military pertaining to boarder area information sharing are major concerns in this research work. Data security concerns have become more common in the management of smart health care since the datasets in health care expand. Therefore, the issue of patient confidentiality seems to be most significant. As a result, improving data security in the advanced medical field seems to be essential in the context of big data and is considered a vital part of a hospital's long-term environmental sustainability. The first challenge in the existing work is the key generation system for encryption and description before transmission and after receiving the data at the receiver. The performance metrics influencing the confidentiality disclosure of big data in the healthcare system was first recognized in this research work, later a model based on risk access control model was developed i.e. store the health care information data in the secured cloud (https://thingspeak.com/) after performing the encryption process with help key generation which is based on the fuzzy theory and Elliptical Curve Cryptography (ECC), which are used in the application of managing big data in smart medical treatments. At last, the results computed by the fuzzy tool as well as the ECC system are verified in MATLAB and are contrasted with the proposed technique. The data protection level is determined with respect to packet delivery ratio, latency, as well as throughput with the help of encrypted as well as decrypted data. From the results obtained, the accuracy can be predicted which is more than $95 \%$ compared to the existing work accuracy of $90 \%$ [1]. The main contribution of this research work for effective key generation is depending on the fuzzy membership sets and ECC

1. With the help of attribute reduction as well as discernibility matrix in the rough set theory, three major features are obtained as well as analyzed on the basis of the factors such as access behavior sensitivity, resource sensitivity, as well as past and present access risk that impact the security of health care in big data:

2. A fuzzy theory-based risk access control framework is introduced and three important benchmarks are fuzzy-treated, as well as the membership function among each index as well as the associated fuzzy set has been calculated.

3. Based on the fuzzy sets which are acting as keys, the encryption and decryption process has been carried out by using holomorphic and Elgamal algorithms for high-level security.

4. The advantages of the ECC-based Elgamal algorithm uses only two computational operations such as point addition and point doubling instead of using computational operations like multiplications, modulo operations, and other complex mathematical operations.

5. During the encryption process, each health care big data is mapping to fuzzy membership sets and then again encrypted by Elgamal methods to increase the security level.

6. The final value of risk is determined by defuzzifying the fuzzy set with the help of the central technique as well as through the inverse process of Elgamal to obtain the final decrypted data which are retrieved from the cloud storage.

7. At last, a comparative study of Elgamal, as well as a fuzzy logic toolset in MATLAB, is carried out to ensure that the framework is effective as well as highly precise.

\section{A. Holomorphic for ECC encryption and decryption}

The important problems discussed in standard differential geometry are the analysis of lengthpreserving, correspondingly, angle-preserving mappings between surfaces in R3. This issue is significant for cartography: the science of graphically indicating a geological area typically on a flat surface for example maps or charts. We realize that there are no length-preserving graphs; but in contrast, anglepreserving mapping exists. The primary objective of the chapter is to illustrate domains in the plane $\mathrm{R}^{2}=$ $\mathrm{C}$ angle-preserving mappings and holomorphic functions are identical. RIEMANN specifically demonstrated the analysis of holomorphic functions for example angle-preserving (= conformal) mappings. It offers the best path to "instinctively realize" such functions. Under such mappings, one can analyze the behaviors of paths. During mapping, the curves intersect each other regularly which leads to invariability of the angles that provide an effective depiction of function. "Conformal mapping corresponding with analytic function provides an outstanding representation of latter's features; it is efficiently contrasted with representation by its graph of a real function."Holomorphic mappings" play an important role in Riemann's function theory. Such mappings present in both domain and range are angle-preserving. A point of issue arises whether the two domains namely $D, D^{\prime}$ are concerned with biholomorphically equivalent, in other words, 
whether a biholomorphically mapping $f: D^{\prime}::^{\prime \prime} D^{\prime}$ exists, although only in few instances it has been resolved, and are effectively verified and found to be successful. Some of the important examples of biholomorphically mappings are described in section 2. Therefore, we will illustrate that a simple function by $\frac{z-i}{z+i}$ which maps the unbounded upper half-plane of $Z$, biholomorphically on the confined unit disc.

A group is formed by the biholomorphic mappings of domain $D$ by itself, referred to as automorphisms group Aut $D$ of $D$. The accurate calculation of automorphisms (usually non-commutative) group is a significant task for Riemann's function theory; except in few cases it is achievable. Section 3 exhibits that automorphisms of both numerator and the unit disk are found among the fractional linear functions $\frac{a z+b}{c z+d}$. These automorphisms are regular at any two points in the region and can be moved by the other. This is referred to as homogeneity and can be utilized for encryption and decryption of ECC and other data. The functions of holomorphic logarithmic (HL) algorithms $\mathrm{H}(\mathrm{z})$ were invented based on the requirements that fulfill the function $e^{H(z)}=z$. Suppose $\mathrm{f}$ is the function of holomorphic in the frequency domain, the function of holomorphic is $\mathrm{h}(\mathrm{p})$ in $\mathrm{f}$ and it satisfies the following equation.

$$
e^{H(z)}=f(z)
$$

Called logarithmic holomorphic $\mathrm{f}$ in $\mathrm{h}$ which is possessed for zero-free in $\mathrm{h}$ and it is proven with assertions for existing holomorphic algorithms by using integrals of contour. It can process the keys generated by ECC for the conversion of signals into integer values so that the length and size of the data will be reduced, to increase the processing time for further processes like features extraction and classifications. The conversion of the ECC key into integer values in the following mathematical model is used over the time interval $\mathrm{T}$.

$$
\frac{1}{2 \pi \gamma} \int f^{\prime}(\delta) / f(\delta) d \delta
$$

The Taylor series of HL for the function of $n$-variable complex operations which is a major role in the creation of local and partial arguments for HL and it is given by

$$
f(p)=\sum_{u \forall m^{n}} \frac{\left(p-p_{0}\right)^{u}}{u !} s^{u} f\left(p_{0}\right), \forall f \epsilon u
$$

It is true for many values of $p_{0} \epsilon h$ and adequately smaller neighborhood $u \forall h$ for all values of $p_{0}$. In this $u \forall m^{n}$ for $\mathrm{n}$-index superscript of power $\mathrm{n}$ and it is given as $\mathrm{n}=\left(\mathrm{n}_{1}, \mathrm{n}_{2}, \mathrm{n}_{3} \ldots, \mathrm{n}_{\mathrm{n}}\right)$ changes from $\mathrm{n}_{1}=\ldots=\mathrm{n}_{2}=0$ to $\mathrm{n}_{1}=\infty$ for all $\mathrm{n}=\left(\mathrm{n}_{1}, \mathrm{n}_{2}, \mathrm{n}_{3} \ldots, \mathrm{n}_{\mathrm{n}}\right) \in n$. This series is efficient and convergent only for signal processing applications pertaining to holomorphic operations.
Suppose $x_{1}=\min \left\{n_{1}\right\}, \ldots \ldots \ldots, x_{n}=$ $\min \left\{n_{m}\right\}$ along with $q^{x} f\left(f_{0}\right) \neq$ nonzero, for the index of $n$ order $\mathrm{x}=$

$\left(x_{1}, \ldots . x_{n}\right)^{t}$ is used for conversion then the proposed equ $f(p)=\left(p-p_{0}\right)^{x} \sum_{u \forall m^{n}} \frac{\left(p-p_{0}\right)^{n-x}}{n !} s^{u} f\left(\left(p_{0}\right)=(p-\right.$ $\left.p_{0}\right)^{x} g(p)$

Where $g(p)$ is perceptibly well-defined and $g\left(p_{0}\right)=$ $\frac{1}{x !} p^{x} f\left(p^{0}\right) \neq 0, \quad \mathrm{~g}(\mathrm{p})$ is holomorphic. And to complete the series, it still has to prove that $\mathrm{x}_{1}>0, \ldots$. $\mathrm{x}_{\mathrm{n}}>0 \quad$ and $\quad\left(p-p_{0}\right)^{x}=$ 1 for all the values of $p$ and $p_{0}$. The above equation is applied for ECG signals to convert into integer values which can apply for further processes in this work.

To realize Elliptic Curve Cryptography (ECC) a good comprehension of basic mathematics is required. The design of ECC broadly depends on conceptual algebra. This chapter thus begins with a summary of the primitive algebraic structures such as groups, rings, and fields. The second portion of this chapter describes the mathematics behind elliptic curves. The research work mainly concentrates on elliptic curves over finite fields of the type $\mathrm{GF}(2 \mathrm{~m})$. Operations are carried out on such form of an elliptic curve. One of the most important characteristics of elliptic curve cryptography is scalar multiplication.

In particular, elliptic curves over finite fields of the type $\mathrm{GF}(2 \mathrm{~m})$ are contemplated. The operations on this type of elliptic curve are conferred. Scalar multiplication is one of the most significant features of elliptic curve cryptography. The sum is given by $\mathrm{nP}$, here the integer is represented as $\mathrm{n}$, and point on an Elliptic curve is given by $\mathrm{P}$ and is defined over a finite field. The sum $\mathrm{nP}$ is specified as follows $[n] p=P+P+\ldots \ldots . .+P$

Are nothing but (n-1) point additions as well as utilizes considerable time in conventional elliptic curve cryptographic structures. Therefore, receive attention with respect to executing elliptic curve cryptosystems. Efficient execution of elliptic curve cryptography relies on:

\section{$>$ Scalar multiplication approach}

$>$ point arithmetic formulae

$>$ Field arithmetic.

The techniques employed in various hierarchical layers portrayed are dependent on each other. This chapter deals with point arithmetic formulae. For specific implementation, the scalar multiplication method is utilized which results in the selection of point arithmetic formulae. Once the scalar $n$ is set, the scalar multiplication approach concentrates on the addition sequence for $\mathrm{n}$ (consider Section 4.1). Yao's method was proposed by Veer Amol Motinath. et.al 
[109] can be utilized, when point $P$ is fixed and precomputation is of most significance. In a typical setting, the familiar Double-and-add algorithm is comparatively the same as that of the square-andmultiply technique which is utilized to calculate $\mathrm{nP}$ by employing 1 number of doublings and approximately $\mathrm{m}$ number of additions where 1 signifies the bit length of $\mathrm{n}$ as well as $m$ represents the hamming weight of $n$. An additional chain for $n$ is produced by the Double-and-Add algorithm. In this work, the Double-as well as-Add algorithm is referred to as binary technique and is further simplified to an m-ary technique. The 2s-ary approach divides the binary depiction of $n$ into $s$ window lengths and a sliding window algorithm that utilizes appropriate pre-computed values that are employed to calculate nP. The benefits of employing public-key cryptography over private key cryptography are the accessibility of improved key administration as well as enhanced protection. On the other hand, because of the complication of basic number-theoretic algorithms, public-key cryptography consumes more time when compared to that of the standard secret-key cryptography, therefore accelerating the requirement for public-key cryptosystems. A mathematical entity known as the elliptic curve is utilized in designing public-key cryptosystems. This thesis concentrates to accelerate elliptic curve cryptography an appropriate substitute for conventional public-key cryptosystems namely RSA. Accelerating ECC is achieved through stepping up point arithmetic algorithms as well as enhancing scalar multiplication algorithms.

This hypothesis offers an acceleration of some point arithmetic algorithms. When a scalar is fixed, the analysis of addition progressions has been carried out and found to be advantageous by enhancing scalar multiplication algorithms. A distinct type of addition chain is known as Lucas chain or a differential addition chain helps perform scalar multiplication on some elliptic curves; for example, Montgomery curves are accessible for various addition formulae. Whereas single scalar multiplication is enough for few systems, there are many other systems where a double or a triple scalar multiplication algorithm is chosen. In this framework, the triple scalar multiplication algorithms for arithmetic chain addition are presented. Pre-computations help to accelerate scalar multiplication algorithms, as soon as the elliptic curve point is set. This research work mainly concentrates on accelerating point computation as well as enhancing scalar multiplication in the framework of pre-computation concerning double scalar multiplication. Additionally, this treatise reexamines pairing calculations that employ elliptic curve sets for determining the pairings, for example, the Tate pairing. Particularly, the hypothesis considers Stange's algorithm for determining pairings as well as pairings on Selmer curves. Also, the thesis examines various features of basic finite field arithmetic.

The achievement of public-key cryptosystems namely Diffie-Hellman key exchange algorithm as well as the ElGamal encryption algorithm mainly depends on a given complexity of DLP in a finite cyclic group, occasionally known as generalized discrete logarithm issue described in the research work of [77], and is defined as Assumed a finite cyclic group $\mathrm{G}$ with a generator a, group order $\mathrm{n}$ as well as some $b € G$, calculate the integer $x, 1 \leq x \leq$ (n - 1) such that $a^{x}=b$. (If $b$ is the identity element in $G$, then $\mathrm{x}=0$ ). In the Diffie-Hellman key exchange algorithm, Alice and Bob aimed at exchanging the private key among them. As a part of the primary operation, a finite cyclic group $G$ is selected with order $\mathrm{n}$ and generator $\mathrm{a}$. Later, Alice selects an integer $\mathrm{x}$ at random $(1 \leq \mathrm{x} \leq(\mathrm{n}-1))$ without disclosing the value of $x$ to anyone, calculates tax, and then transfers it to Bob through an open channel. Bob selects an integer $\mathrm{y}$ at random $(1 \leq \mathrm{y} \leq(\mathrm{n}-1))$ without disclosing the value of $\mathrm{y}$ to anyone computes $\mathrm{a}^{\mathrm{y}}$ and sends it to Alice. $\left(a^{y}\right)^{x}$ is computed by Alice and $\left(a^{x}\right)^{y}$ is computed by Bob, hence ensuing both Alice and Bob share the private key $\mathrm{a}^{\mathrm{xy}}$ among themselves. Interloper Eve can retrieve both $\mathrm{a}^{\mathrm{x}}$ and $\mathrm{a}^{\mathrm{y}}$, possibly will calculate $\mathrm{a}^{\mathrm{xy}}$. The difficulty encountered by Eve is often known as the GDHP in the literature proposed by Dominique. et.al. [39].

A well-organized algorithm to deal with DLP will allow Eve to figure out the GDHP, it can determine $\mathrm{x}, \mathrm{y}$ and thus calculate $\mathrm{a}^{\mathrm{xy}}$, ensuing arbitrating of the cryptosystem. An efficient algorithm to resolve DLP deals with the Diffie-Hellman key exchange algorithm as well as deals with cryptosystems like the ElGamal encryption system. It is assumed that the complexity in solving the GDHP is equal to the complexity in solving DLP.

\section{B. Affine Coordinates}

If $\mathrm{X}=\left(\mathrm{p}_{1} ; \mathrm{q}_{1}\right)$ and $\mathrm{Y}=\left(\mathrm{p}_{2} ; \mathrm{q}_{2}\right)$ are the two distinct points on $E, X \neq O ; Y \neq O$ as well as $X \neq \pm Y$, then $X+$ $\mathrm{Y}=\left(\mathrm{p}_{3} ; \mathrm{q}_{3}\right)$ is given by

$$
\begin{gathered}
p_{3}=\alpha^{2}-p_{1}-p_{2} \\
q_{3}=\alpha\left(p_{1}-p_{3}\right)-q_{1}
\end{gathered}
$$

Where if $\mathrm{X}=\mathrm{Y}=\left(\mathrm{p}_{1}, \mathrm{q}_{1}\right)$ and $\mathrm{X} \neq-\mathrm{X}$ and $2 \mathrm{X}=\left(\mathrm{p}_{3}, \mathrm{q}_{3}\right)$ is defined as

$$
\begin{gathered}
p_{3}=\propto^{2}-2 p_{1} \\
q_{3}=\propto\left(p_{1}-p_{3}\right)-q_{1}
\end{gathered}
$$


The value of one inversion is considered as $\mathrm{C}$, the value of one multiplication is taken as $\mathrm{M}$ and $\mathrm{S}$ is the value of one square in $\mathrm{K}$, then $1 \mathrm{C}+2 \mathrm{M}+1 \mathrm{~S}$ operations are required for several additions, and $1 \mathrm{C}+2 \mathrm{M}+2 \mathrm{~S}$ operations are required for several doublings. On the other hand, the value of the field operations is expressed as $\mathrm{C} ; \mathrm{M}$ and $\mathrm{S}$ are measured, whereas the value of a field addition or filed subtraction is neglected as it is small when compared to that of $\mathrm{M}=\mathrm{S}=\mathrm{C}$. However, squaring is distinctly a unique case of multiplication, where the operands are identical; differentiating between these two operations is useful because it is possible to alter squaring by offering significant acceleration over a multiplication. 2 is the best achievable acceleration factor. An effective thumb rule considers the value of squaring as approximately $2=3$ times of multiplication [17]. These similarities rely on the basic implementation of these operations. In some applications, $\mathrm{S}=0: 8 \mathrm{M}$. To determine the inverse $\mathrm{K}$ is an important function which is usually $\mathrm{C}>30 \mathrm{M}$. We signify the value of multiplication by a small constant $\mathrm{c}$ as Mc. This is suitable for differentiating $\mathrm{M}$ as well as Mc as special-purpose algorithms and is utilized for constant multiplication. Many approaches from various study areas and also researchers, endeavors have failed to provide data security, programmed data, special characters, and images [9-33]. Key size is a fundamental parameter for all applications if the key size is large, the security level is high [10]. There are many encryption and decryption computations, for example, $\mathrm{BCH}$, hamming codes, piece codes such as LDPC, AES, as well as RS codes and among these codes, the AES employs excessive key size i.e. 256 bits and other computations, utilizes the key size of just 8 bits [33]. A most important challenging framework has been developed at that point which provides high security and key size termed as Elliptic Curve Cryptography (ECC) since this framework achieves high security with the help of resources [11]. In the existing work, a new concept is proposed to develop a typical framework that provides a greater level of security for various types of data. In this design, ECC has been fine-tuned to 256 unique keys sizes to form a $16 \times 16$ framework and each key size is 32 bits which include two distinctive characters known by the transmitter and receiver. The unique character combines the $\mathrm{x}$ and $\mathrm{y}$ plans given in equation (1).

$$
\mathrm{Key}_{\text {special characters }}=(\mathrm{x} \& * \mathrm{y})
$$

Here the special character of the transmitter is represented by $\&$ and the special character of the receiver is represented by $*$.

The design is highly protected and the less complex system comprises, the cloud architecture, system handling for complexity and management of cloud access control. This system is performed for multiple users with various cloud models with high security and recovery of the data, segmentation of data is discussed in [32]. As depicted in Fig.1. It has several phases of process which is described below:

$>$ Creation of account in a cloud by using personal information along with the secret key.

$>$ Obtaining biomedical signals and images such as EEG/ECG and Endoscope Ultrasound Images.

$>$ By employing the Holomorphic algorithm, the signals and images are converted into integer format.

$>$ Encryption through ECC- ElGamal algorithm.

$>$ The data is stored in the cloud via different channel IDs and passwords.

$>$ Access policy authentication in a cloud server.

$>$ Decryption by ECC- ElGamal algorithm

In the ECC cryptography context, the new algorithm facilitates a limited form of elliptic curve considered over a limited field (ffp). Essential number $p$ is referred to as "mod p", it is an elliptic grouping as well as p consistently is a prime number and it is illustrated in equation (2).

$$
\left(4 G_{1}^{3}+27 G_{2}^{2}\right) \bmod p \neq 0
$$

The elliptic group is represented by $E_{p}$ and mod $p$ of coordinates $(\mathrm{x}, \mathrm{y})$ is the pair of positive integers not greater than $\mathrm{p}$ and must satisfy the equation (3)

$y^{3}=\left(x^{3}+G_{1} x+G_{2}\right) \bmod p$

The $E_{p}\left(G_{1}, G_{2}\right)$ group consists of several points as well as all special characters and infinity $(\Omega)$.

\section{Generation of points on the curve}

To satisfy the eq (2) as well as eq (3), the values $\mathrm{G} 1=1$ also $\mathrm{G} 2=1$ are chosen. A network of $16 \times 16$ is established by considering 256 total focal points, for which $\mathrm{p}=463$ is selected, as stated by ECC control, as $p$ values increases, gradually there is an increase in the security of the defined work $[12,21]$. The value of $\mathrm{p}$ goes from 1 to 463 as well as replaces all the numbers in eq's ( 3 and 4 ) on the left-hand side as well as right-hand sides. The left-hand side condition is addressed in eq (4) and the right-hand side condition is specified in eq (5).

$$
\begin{aligned}
& y_{\text {coordinate }}=y^{2} \bmod p \\
& x_{\text {coordinate }}=\left(x^{3}+G_{1} x+G_{2}\right) \bmod p
\end{aligned}
$$

The computed left-hand side, as well as right-handside values, are tabulated in Annexure-4.1. From Annexure-4.1, the coordinated directions point (x, y) are known, for $\mathrm{p}=463$ there are only 10 coordinated 
focal points and those coordinated are recognized. One of the coordinated focal points is preferred as the initial point, i.e. $\mathrm{P}=(70,86)$, and make use of this basic point, the following focuses such as $2 p, 3 p, 4 p, 5 p, \ldots \ldots, 256 p$ are calculated by using point addition and point doubling. The figured 256 focuses are noted in Table.1. The advantages of Point Addition, as well as Point Doubling, is to reduce the number of arithmetic operations as well as scalar addition i.e $\mathrm{kp}$ where $\mathrm{k}$ is constant and changes from 1 to 463. The Point Addition, as well as Point Doubling constraints, are mentioned in eq (6) as well as in eq (7).

EC operation on Prime Field $F_{p}$ : An elliptic curve $E$ over prime field $F_{p}$ is defined as the set of points $\mathrm{A}\left(\mathrm{x}_{\mathrm{A}}, \mathrm{y}_{\mathrm{B}}\right)$ with $\mathrm{x}, \mathrm{y} \in \mathrm{F}_{\mathrm{p}}$ which fulfills Weierstrass equation-

$$
y^{2}=a x+b(\bmod p)
$$

Where curve parameters are represented by $\mathrm{a}$ and $\mathrm{b}$ that belongs to $F_{p}$. The discriminator equation is specified by $4 a^{3}+27 b^{2} \neq 0$. $\mathrm{p}$ is the field parameter and field size is given by the length of the bits.

\section{Point Addition as well as Point Doubling on $F_{p}$ :}

In the case of point addition, the addition is achieved over two points on the elliptic curve with various $x$ coordinates. Let us assume those two points be $\mathrm{A}\left(\mathrm{x}_{\mathrm{A}}\right.$, $\left.\mathrm{y}_{\mathrm{A}}\right)$ and $\mathrm{B}\left(\mathrm{x}_{\mathrm{B}}, \mathrm{y}_{\mathrm{B}}\right)$ on the $\mathrm{EC}(\mathrm{A} \neq B)$, a line is drawn from point $A$ to point $B$. This line, when extended intersects the elliptic curve at a third point $-\mathrm{C}$. Further, the point $-\mathrm{C}$ is reproduced in the $\mathrm{x}$-direction for acquiring point $\mathrm{C}$ as represented in Figure. 4.5(a $\& \mathrm{~b})$ which indicates the resulting point addition operation $A+B=C$. If $A=-B$, then extending a line by joining the points $A$ and $B$ provides a vertical line that is extended to meet the point at infinity. On the other side (Figure 4.6). Point Doubling operation is the recurring method of adding point $\mathrm{A}$ to itself (i.e., $A+A=2 A=C$ ). This operation is performed by drawing a tangential line to $\mathrm{EC}$ at point $\mathrm{A}$ and which meets the elliptic curve at point C. By reproducing the point at $\mathrm{C}$ in $\mathrm{X}$-direction point $\mathrm{C}$ can be attained.

In general, the security level is various for different types of databases, and several research works have been carried out for improving the security level for binary data which are using in $\mathrm{NoC}$, SoC, and digital communications and also there is some research work that can provide security for more informative images like endoscopy images and biomedical signals such as ECG as well as EEG. In the literature survey, it is found that security can be provided for the individual database at different levels of security but there is no single system that can provide more security to all databases at a time like binary, images, ECG, and
EEG together. The proposed research work, certainly excellent security has been provided for all databases formally introduce as hybrid holomorphic based encryption and decryption (HHED) algorithm. This algorithm is the combination of effective key generation by ECC, high-security AES algorithm, accurate conversion from any data into integer values by holomorphic algorithm, and storage system i.e. cloud. The holomorphic based hybrid high-security system has three different distinct, out of which, the first stage for binary encryption and decryption which is done after conversion into an integer by using a holomorphic algorithm, the second stage is based on holomorphic with AES for endoscopy images, the third stage is for ECG/EEG Signal encryption and decryption and finally, all encipher information is stored in the cloud through RTP and RTCP protocol. The authorized people can download the data and then apply the decryption for the reconstruction of the original database as shown in Fig.1.

The proposed hybrid Fuzzy, holomorphic, and Elgamal techniques are investigated for different databases for various noisy conditions under the transmission of data through the RTP-RTCP protocol. For every MSE and Packet Delivery Ratio (PRD) value, the SVM-based machine learning algorithm is evaluating the system performance like whether MSE and PRD are optimized or not. The selection of the MSE reduction technique is purely based on threshold boundary values assigned to it. The selection of schemes like Fuzzy or ECC using SVM classifier improves the performance level of the data privacy system and it also reduces the complexity trade-off measurement as shown in Fig.1 The threshold boundary assigned to the system are $[6,7,8,9]$ and for every boundary value, the MSE reduction is evaluated by the SVM technique and its step-step process is shown in below Machine learning algorithm. From step 1 to 3 are to select boundary values and its training the network based on the excepted MSE and PRD values (predefined values). Based on the PRD and MSE values, the multi-rate SVM is evaluating and select either Fuzzy or ECC techniques for further reduction of MSE and PRD.

\section{E. Point Addition (PA)}

Let $\mathrm{p}=\left(\mathrm{x}_{1}, \mathrm{y}_{1}\right)$ and $\mathrm{q}=\left(\mathrm{x} 2, \mathrm{y}_{2}\right)$, both $\mathrm{p} \& \mathrm{q}$ are belongs to $\mathrm{ff}_{\mathrm{p}}$ then $\mathrm{p}+\mathrm{q}=\left(\mathrm{x} 3, \mathrm{y}_{3}\right)$

Where

$$
\begin{gathered}
x_{3}=\left[-x_{1}-x_{2}+\left(\frac{y_{2}-y_{1}}{x_{2}-x_{1}}\right)^{2}\right] \bmod p \\
y_{3}=\left[-y_{1}+\left(\frac{y_{2}-y_{1}}{x_{2}-x_{1}}\right)\left(x_{1}-x_{3}\right)\right] \bmod p
\end{gathered}
$$




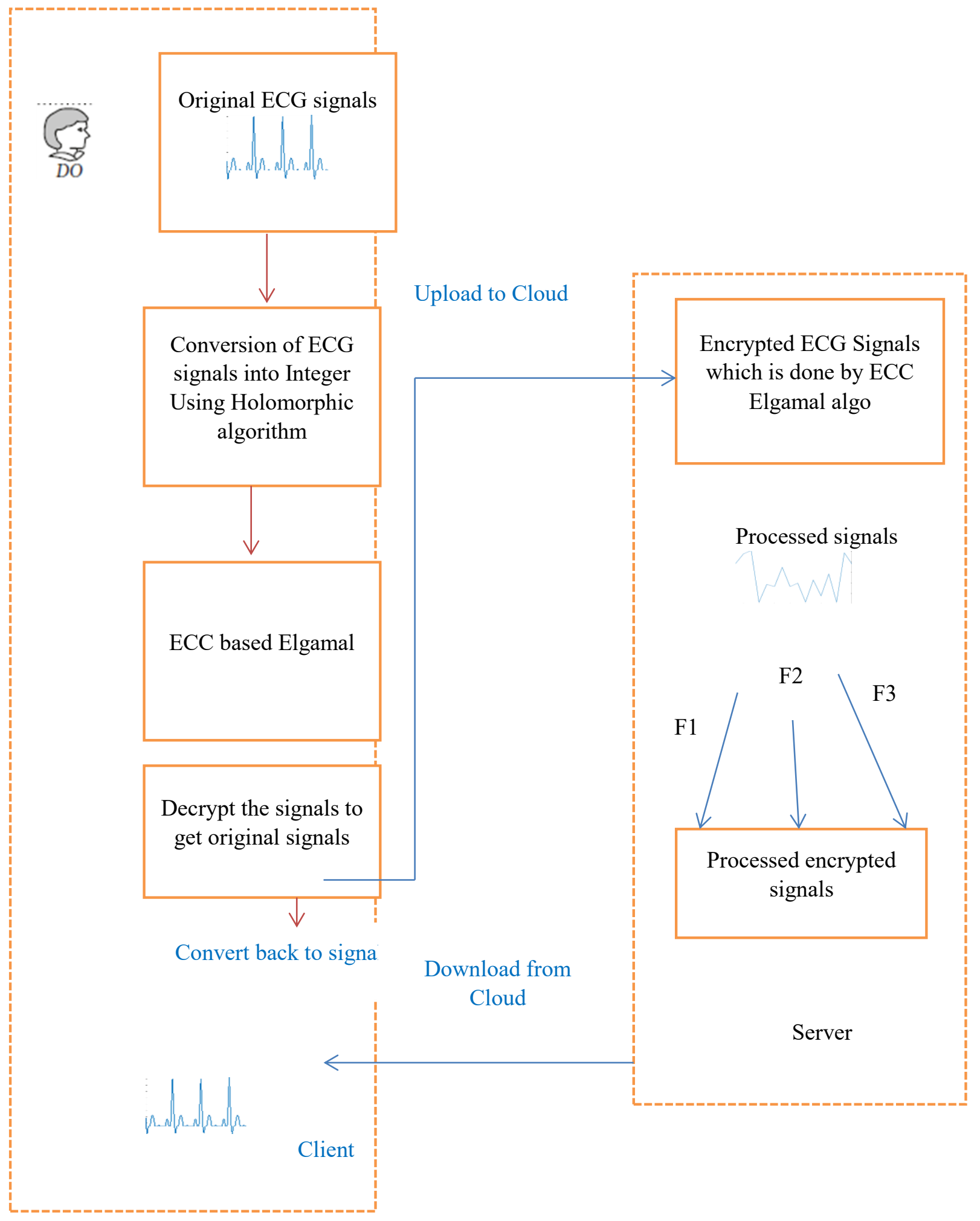

Fig.1 Overall proposed block diagram of secured and storage systems for biomedical signals and biomedical images.

To add the 2 points using point addition, for example $\mathrm{P}=(16,8)$ and $\mathrm{Q}=(19,28)$

$$
\begin{aligned}
& \lambda=\left(\mathrm{y}_{2}-\mathrm{y}_{1}\right) /\left(\mathrm{x}_{2}-\mathrm{x}_{1}\right)=20 / 3=20 * 21 \bmod 31 \\
= & 17
\end{aligned}
$$

$$
\begin{aligned}
& \lambda=17 \\
& \mathrm{x}_{3}=\lambda^{2}-\mathrm{x}_{1}-\mathrm{x}_{2}=17^{2}-19-16=254 \bmod \\
& 31=6 \\
& \mathrm{y}_{3}=\lambda\left(\mathrm{x}_{1}-\mathrm{x}_{3}\right)-\mathrm{y}_{1}=17(16-6)-8=162 \\
& \bmod 31=7
\end{aligned}
$$


Hence $\mathrm{P}+\mathrm{Q}=\left(\mathrm{x}_{3}, \mathrm{y}_{3}\right)$ i.e. $(16,8)+(19,28)$ $=(6,7)$

$$
\text { i.e. } 9 \mathrm{P}+18 \mathrm{P}=27 \mathrm{P} \text {. }
$$

To add the 2 points using point doubling $\mathrm{P}=(18,29)$

$$
\lambda=\left(3 \mathrm{x}_{1}^{2}+\mathrm{a}_{1}\right) /\left(2 \mathrm{y}_{1}\right)=\left(3 * 18^{2}+1\right) /(2 * 29)=
$$
$12 / 27=12 * 23 \bmod 31=28$

$$
\begin{aligned}
& \lambda=28 \\
& \mathrm{x}_{3}=\lambda^{2}-\mathrm{x}_{1}-\mathrm{x}_{2}=28^{2}-18-18=748 \mathrm{mod} \\
& 31=4 \\
& \mathrm{y}_{3}=\lambda\left(\mathrm{x}_{1}-\mathrm{x}_{2}\right)-\mathrm{y}_{1}=28(18-4)-29=363 \\
& \text { mod } 31=22 \\
& \text { Hence } 2(18,29)=(4,22) \\
& \text { i.e. } 2 \mathrm{P}+2 \mathrm{P}=4 \mathrm{P} .
\end{aligned}
$$

Since asymmetric key cryptography is essential for communication, there is a major drawback in the application's performance. Complex mathematical computations are the main drawback of most publickey cryptographic algorithms. Thus, significant implementations of the algorithms are efficient. To develop efficient cryptographic implementations there exist mainly two schemes. In software platforms, the first and foremost scheme concentrates on executing and enhancing cryptographic algorithms. The benefits of this scheme are that it costs very low and it does not require any extra hardware. On the other hand, the advantages achieved by this scheme are constrained by the architecture confines of the microprocessor. In the case of microprocessors, computations on large numbers are not executed efficiently as they can be achieved on customized hardware. Such computations are a standard in public-key cryptographic algorithms. Also, the software can easily be manipulated and therefore providing security for the application. Though software executions are customized to utilize the processor's architecture [36-37] they do not match the given hardware implementations. The intrinsic parallelism, flexibility, and standard design of hardware Table.1 Generated 256 points through Point Addition and Point Doubling on ECC curve considerably accelerate the implementation. In contrast to software, hardware devices can be easily modified. This is advantageous for cryptographic applications. In contrast, the software is less expensive than hardware and offers a limited number of resources. Design procedures for hardware are more complicated and memory is another limitation for such schemes. It is thus essential to have compressed, scalable, and integrated hardware designs that are modified to the given application. Field programmable gate arrays are reconfigurable platforms to create hardware. Uses of hardware platforms, as well as software platforms, are presented. Also, they present more programmability and software platform of less cost, similarly, hardware employment affords better performance than a software implementation. However, designing FPGAs is more complex. A design for an applicationspecific integrated circuit (ASIC) library always does not function for an FPGA. The variations occur because of the characteristic difference in the libraries and the architectures. FPGAs possess constant resources, a lookup table (LUT) based architecture, and larger integrated delays. Later modeled FPGA must be precisely constructed to make use of the resources properly and fulfill the timing constraints of the FPGA library. In this proposed work a sidechannel attack (SCA) against the elliptic curve processor on an FPGA platform is designed and implemented. Meanwhile, in affine coordinates, one inversion operation is required for every addition or a doubling, depending on the number of point additions or doublings that is essential, the number of inversions increases. The above drawback is avoided by employing projective coordinates, but the outlay of extra memory, Z-coordinates for all points being handled must be stored.

Where $\left(\frac{y_{2}-y_{1}}{x_{2}-x_{1}}\right)$ is specified as $\lambda$ as well as when $\lambda$ is non-positive then the following unique cases are taken into account to fulfill the basic condition of ECC as shown in Fig.3.

\begin{tabular}{|l|l|l|l|l|l|l|l|l|}
\hline Points & \multicolumn{2}{|l|}{ Points on the elliptic curve } \\
\hline $1-8$ & $(70,86)$ & $(270,328)$ & $(226,429)$ & $(104,86)$ & $(294,324)$ & $(73,287)$ & $(179,19)$ & $(390,302)$ \\
\hline $9-16$ & $(182,394)$ & $(299,334)$ & $(82,271)$ & $(304,401)$ & $(223,82)$ & $(292,148)$ & $(268,134)$ & $(373,216)$ \\
\hline $17-24$ & $(8,153)$ & $(321,232)$ & $(283,100)$ & $(256,280)$ & $(258,161)$ & $(122,327)$ & $(68,226)$ & $(235,354)$ \\
\hline $25-32$ & $(102,36)$ & $(132,197)$ & $(392,383)$ & $(244,241)$ & $(95,384)$ & $(137,59)$ & $(438,394)$ & $(34,294)$ \\
\hline $33-40$ & $(78,166)$ & $(139,377)$ & $(137,336)$ & $(449,340)$ & $(8,410)$ & $(90,109)$ & $(324,409)$ & $(14,150)$ \\
\hline
\end{tabular}


INTERNATIONAL JOURNAL OF BIOLOGY AND BIOMEDICAL ENGINEERING

\begin{tabular}{|c|c|c|c|c|c|c|c|c|}
\hline $41-48$ & $(437,135)$ & $(334,59)$ & $(237,10)$ & $(263,414)$ & $(246,289)$ & $(430,454)$ & $(374,200)$ & $(8,273)$ \\
\hline $49-56$ & $(228,286)$ & $(253,407)$ & $(296,269)$ & $(428,80)$ & $(236,222)$ & $(339,6)$ & $(217,315)$ & $(304,391)$ \\
\hline $57-64$ & $(157,412)$ & $(432,204)$ & $(251,274)$ & $(15,11)$ & $(24,103)$ & $(442,132)$ & $(112,327)$ & $(126,378)$ \\
\hline $65-72$ & $(306,337)$ & $(73,53)$ & $(441,291)$ & $(355,406)$ & $(87,33)$ & $(178,115)$ & $(73,53)$ & $(110,452)$ \\
\hline $73-80$ & $(16,84)$ & $(424,261)$ & $(253,73)$ & $(162,144)$ & $(328,293)$ & $(247,165)$ & $(276,402)$ & $(13,93)$ \\
\hline $81-88$ & $(452,275)$ & $(358,123)$ & $(166,56)$ & $(417,225)$ & $(117,365)$ & $(175,197)$ & $(225,44)$ & $(433,96)$ \\
\hline $89-96$ & $(29,279)$ & $(157,380)$ & $(330,137)$ & $(206,158)$ & $(29,451)$ & $(10,80)$ & $(258,173)$ & $(139,52)$ \\
\hline $97-104$ & $(126,102)$ & $(97,237)$ & $(67,144)$ & $(53,64)$ & $(284,209)$ & $(203,108)$ & $(83,347)$ & $(272,346)$ \\
\hline $105-112$ & $(13,267)$ & $(366,131)$ & $(328,258)$ & $(14,260)$ & $(162,51)$ & $(187,34)$ & $(374,154)$ & $(14,24)$ \\
\hline $113-120$ & $(69,97)$ & $(445,335)$ & $(186,367)$ & $(56,371)$ & $(64,321)$ & $(384,20)$ & $(343,225)$ & $(154,259)$ \\
\hline $121-128$ & $(79,375)$ & $(328,147)$ & $(214,121)$ & $(31,454)$ & $(27,458)$ & $(75,108)$ & $(82,56)$ & $(239,162)$ \\
\hline $129-136$ & $(239,162)$ & $(376,12)$ & $(82,14)$ & $(347,187)$ & $(306,195)$ & $(205,348)$ & $(321,298)$ & $(73,380)$ \\
\hline $137-144$ & $(59,348)$ & $(423,72)$ & $(299,162)$ & $(407,158)$ & $(125,376)$ & $(93,382)$ & $(437,4)$ & $(182,127)$ \\
\hline $145-152$ & $(399,134)$ & $(315,292)$ & $(93,369)$ & $(370,128)$ & $(241,66)$ & $(209,266)$ & $(188,434)$ & $(308,452)$ \\
\hline $153-160$ & $(146,289)$ & $(428,164)$ & $(318,58)$ & $(330,257)$ & $(69,351)$ & $(173,355)$ & $(282,395)$ & $(58,80)$ \\
\hline $161-168$ & $(451,418)$ & $(11,172)$ & $(81,189)$ & $(128,339)$ & $(132,441)$ & $(384,222)$ & $(212,225)$ & $(230,155)$ \\
\hline $169-176$ & $(37,17)$ & $(104,348)$ & $(162,440)$ & $(155,60)$ & $(217,199)$ & $(357,443)$ & $(167,166)$ & $(415,160)$ \\
\hline $177-184$ & $(419,280)$ & $(282,356)$ & $(175,78)$ & $(31,83)$ & $(36,304)$ & $(4,19)$ & $(234,274)$ & $(295,232)$ \\
\hline $185-192$ & $(441,334)$ & $(303,142)$ & $(444,412)$ & $(204,332)$ & $(62,136)$ & $(399,234)$ & $(150,333)$ & $(329,104)$ \\
\hline $193-200$ & $(179,205)$ & $(286,18)$ & $(44,334)$ & $(303,142)$ & $(444,412)$ & $(204,332)$ & $(62,136)$ & $(399,234)$ \\
\hline $201-208$ & $(107,275)$ & $(51,424)$ & $(455,67)$ & $(143,9)$ & $(334,89)$ & $(347,95)$ & $(271,6)$ & $(21,293)$ \\
\hline $209-216$ & $(97,50)$ & $(187,34)$ & $(186,281)$ & $(225,320)$ & $(211,427)$ & $(387,59)$ & $(3,81)$ & $(334,226)$ \\
\hline $217-224$ & $(33,165)$ & $(210,288)$ & $(154,259)$ & $(79,375)$ & $(154,122)$ & $(88,237)$ & $(202,258)$ & $(66,284)$ \\
\hline $225-232$ & $(401,183)$ & $(81,115)$ & $(384,391)$ & $(373,267)$ & $(369,152)$ & $(369,152)$ & $(260,397)$ & $(15,206)$ \\
\hline $233-240$ & $(157,188)$ & $(360,150)$ & $(92,254)$ & $(127,347)$ & $(42,359)$ & $(217,118)$ & $(218,181)$ & $(135,287)$ \\
\hline $241-248$ & $(292,182)$ & $(19,317)$ & $(151,63)$ & $(14,31)$ & $(262,453)$ & $(38,407)$ & $(145,131)$ & $(173,438)$ \\
\hline $249-256$ & $(456,312)$ & $(249,337)$ & $(366,143)$ & $(413,354)$ & $(395,254)$ & $(42,313)$ & $(337,177)$ & $(199,64)$ \\
\hline
\end{tabular}

(1) If the upper part of the fraction is non-positive then any one of the following processes can be employed.

i. For the non-positive upper part of the fractional value, perform modulo operation (or)

ii. Obtain the numerator and then employ signed multiplication
(2) If the lower part of the fraction is non-positive then any one of the following processes can be employed

i. For the numerator value that is inverse, perform modulo operation (otherwise)

ii. Consider the numerator value and carry out signed multiplication 
(3) If denominator, as well as numerator, are nonpositive then either of the following processes can be applied

i. For the upper half of the fraction as well as the lower half of the fractional values, perform modulo operation (otherwise) ii. Determine the denominator and further multiply the denominator with the upper part of the fraction.
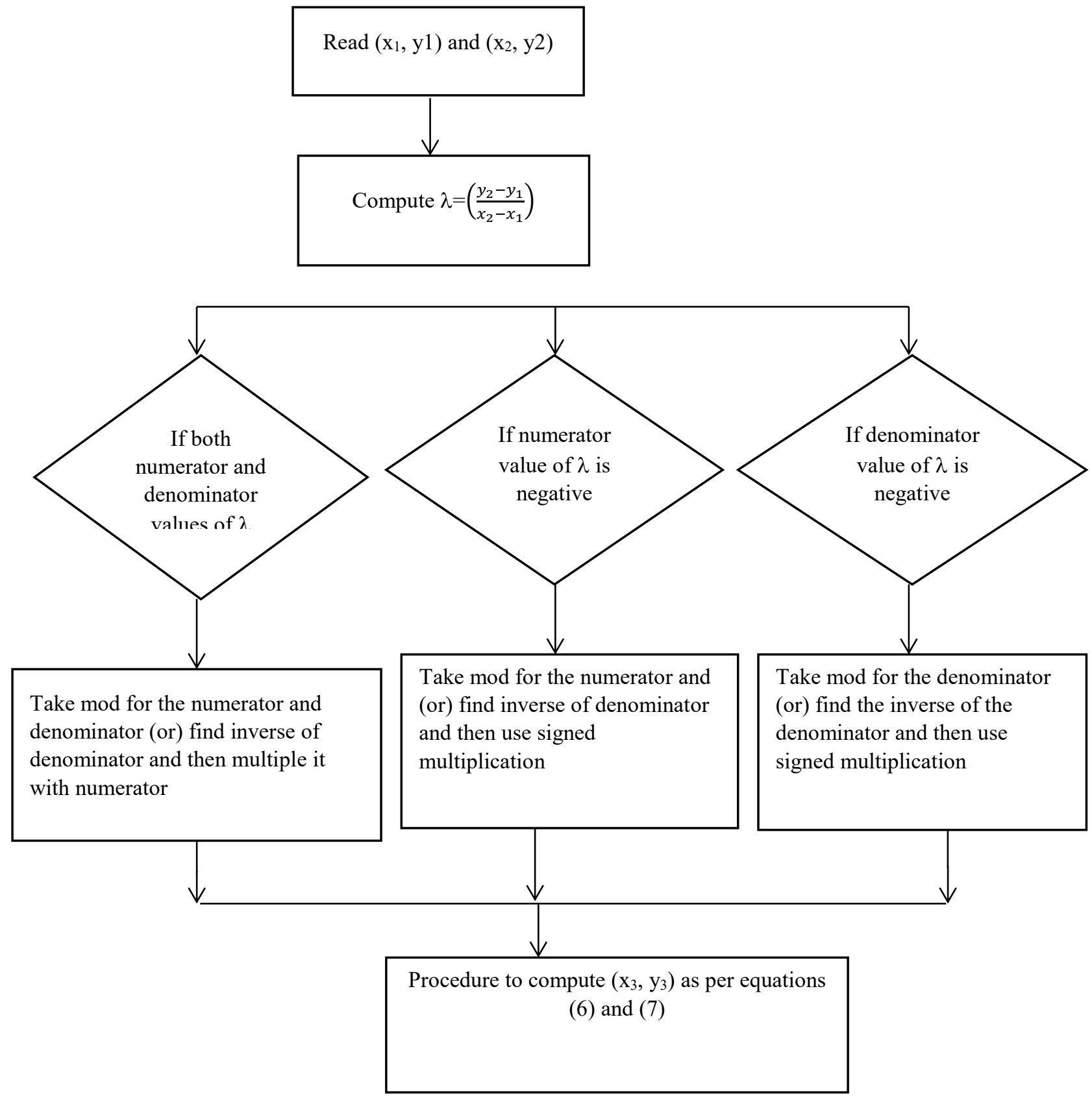

Fig. 2 Flow chart for the finding of sign in $\lambda$

The following special cases are considered to meet the requirements of ECC when $\left(\left(y \_2-y \_1\right) /\left(x \_2-x \_1\right.\right.$ ) ) is specified as $\lambda$ and if $\lambda$ is specified as negative. One of the following criteria can be used if the upper part of the fraction is not $\mathrm{n}$ positive as shown in Fig.2.

Perform a modulo operation (or) on the upper value of the fraction that is not positive
One of the following criteria can be used if the lower part of the fraction is negative.

Perform modulo operation (or) for the lower part of the fraction.

Signed multiplication is carried out by considering the upper value of the fraction. One 
of the above-listed procedures can be used if both are negative.

$>$ Carry out modulo operations (or) on the upper as well lower parts of the fractional values. After the above step, multiply the numerator by the fraction's upper half.

\section{F. Point doubling (PD)}

Point Doubling is used to generate point doubling when the input is single point, consider an instance $2 p=p+p, \quad 4 p=2 p+2 p, \quad 8 p=4 p+4 p, \quad$ etc. The implementation of PD on single point say $p, 2 p, 4 p \ldots$, etc., are carried out by considering $\mathrm{p}=\left(\mathrm{x}_{1}, \mathrm{y}_{1}\right)$ then $2 \mathrm{p}=\left(\mathrm{x}_{3}, \mathrm{y}_{3}\right)$, where

$x_{3}=\left[-2 x_{1}+\frac{3 x_{1}-G 1}{2 y_{1}^{2}}\right] \bmod p \quad$ and $\quad y_{3}=\left[-y_{1}+\right.$ $\left.\frac{3 x_{1}-G 1}{2 y_{1}^{2}}\right] \bmod p$

By using Point Addition and Point Doubling, 256 focal points are generated and the same focal points and additionally. Each point enables $\mathrm{x}$ as well as $\mathrm{y}$. Therefore, to achieve smooth functioning through highly-developed processors, the $\mathrm{x}$, as well as $\mathrm{y}$ coordinates, are combined into a single by inserting two special characters between them. Each coordinate is 8-bit in size and the size of a special character is 8 bits, the total size of the point is 32 bits which is given in equation (8).

$$
\mathrm{Key}_{\text {special characters }}=(70 \& * 86)=(\mathrm{x} \& * \mathrm{y})
$$

1000110,1010110 is the binary representation of 70 as well as 86 respectively, and 00100110, 00101010 is the binary representation of and as well as * respectively. Therefore equation (8) may be represented as follows: Key special characters $=100011010101100010011000101010$.

The binary data which is of 32 bit is given as input to the encipher-texting. Once the computations of 256 focal points using point addition and point doubling have been carried out successfully, every focal point is stored in Look-Up-Table and depends on the 8-bit data input; further, the 32 bits of LUT value is been selected. The 32-bit Look-Up-Table value is transmitted through Routers or Network Interface. To reduce power dissipations in NI before transmitting the data via a communication channel, then data encryption is performed; the obtained encoded information provides less security. In the proposed work, we present three approaches to reduce the number of conversions; each approach is an advanced version of the previous method. The basic methods can reduce just $25 \%$ of improvements, Partial developments decline in subsequent approach and $85 \%$ reduction in the last approach. The three approaches are analyzed regarding power dissipation which is associated with many variations.

\section{G. Novel Public-Key Encryption}

From point addition and point doubling the generated point is encrypted by utilizing the ECC- Elgamal algorithm for all 256 elliptical points to enhance the security level. In the proposed encryption, before transmission through a protocol, the power consumption is reduced and stored in the cloud. The encipher-texting process of ECC- Elgamal algorithm is as follows:

A finite cyclic group $G$ is set i.e. $G=\left(Z_{p}\right)^{*}$ which is of $n$ order for the fixed generator $g$ in $G$ i.e $G=\{1$, $\left.\mathrm{g}, \mathrm{g}^{2}, \mathrm{~g}^{3}, \ldots, \mathrm{g}^{\mathrm{n}-1}\right\}$. For encipher-texting, the private key is the output of equation (8) which is utilized by the sender and the same key used for the receiver. The encryption process is given in equation (10)

$E_{p}=\operatorname{data}^{G} \quad----(10)$ and

the ciphertext is $c_{t}=E_{p} *$ Key special characters------------(12)

By utilizing Eqn (11) and (12), from point addition and point doubling the original data is encrypted for all generated points and resultant values are uploaded into the cloud for storage.

\section{H. Novel Public-Key Decryption}

The data from the cloud is encrypted and read and decrypted by employing the ECC-Elgamal algorithm which uses an identical symmetric key. The decrypted data is examined to compute the simulation time, throughput, and processing time.

\section{Decryption process}

Input: Data from the cloud which encrypted by the ECC-Elgamal algorithm and Key special characters

Output: Reconstructed data

Step-1 Read secret key form the memory

Step-2 Compute $\mathrm{G}^{\mathrm{g}}$ and derive symmetric key $\mathrm{k}$ and compute $\mathrm{c}=\mathrm{kg}$ Key special characters

\section{Step-2 Perform $\quad \mathrm{G}^{\mathrm{g}}-\mathrm{kg}$ Key special characters \\ Step-3 Calculate throughput in $\mathrm{GHz}$}

\section{RESULTS AND DISCUSSION}

Encryption is a process of converting the original information into integer information to enhance the security level in this research work. The ECC with Elgamal is a highly-developed method for more efficient and reduced mathematical operations in cloud security, which provides protection depending on the rigidity of various problems. This system put 
up corresponding security with limited expenditure. The characteristics of this designed algorithm are, it completely depends on the key and in order values are depicted in Table.1. Furthermore, it provides improved response for the original information, and it enables the transmission of keys securely between the sender and receivers. In general, the ECCHolomorphic-Elgamal algorithm carries out arithmetic operations such as Point doubling and point addition on the information and, encrypted data performs some addition and modulo operations. Additionally, it is the most suitable solution for protecting the original information security issues in the cloud. The important benefits of these modern systems are rapid calculation and security enhancement.

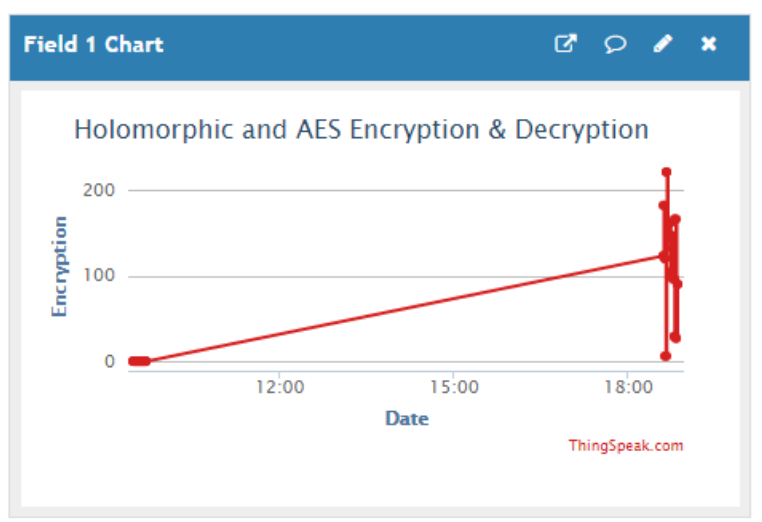

Hence, the ECCHE system is realized in this research work to confidentially allocate the cloud data. In this work, the private Key exceptional characters are shown in eq (8) and input information i.e. EEG/ECG and Image are applied as input and are depicted in Fig.3. Afterward, by using the encryption process the Ciphertext is created, and the information is encrypted via Elgamal, generated public key, and point on the ECC curve. Later, the Holomorphic process is given to the encrypted information which is reliant on point doubling and points addition property. Furthermore, three constants, for example, each encrypted information $\mathrm{x}, \mathrm{y}, \mathrm{z}$ are processed, finally, the encrypted information is passed on and is uploaded on cloud and uploaded information are in the form of .csv and interlaced as a graph and is represented in Fig.4.

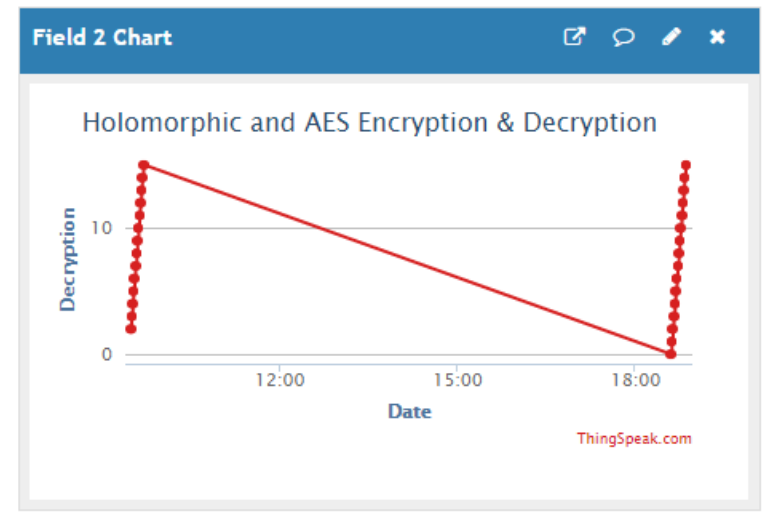

Fig.3 Encrypted and decryption of data storage in Cloud in form of a graph

Once the stored data is downloaded from the cloud through the application process, the ECC-Elgamal decryption process is executed to get back the original data. In this decryption phase, the ciphertext values are obtained from the cloud in which data is already encrypted by ECC and Reverse process of Elgamal process, thereafter the ECC through decrypted Elgamal algorithm is applied to transform into original and by using the same secret key employed for encryption process the data is mapped in EEG/ECG and Image format. As soon as the data is accessed from the cloud, decryption of data is done through a point on a curve, and the Holomorphic operations are applied to the data for producing the original text as displayed in Fig.5. 


\section{AES_holomorphic}

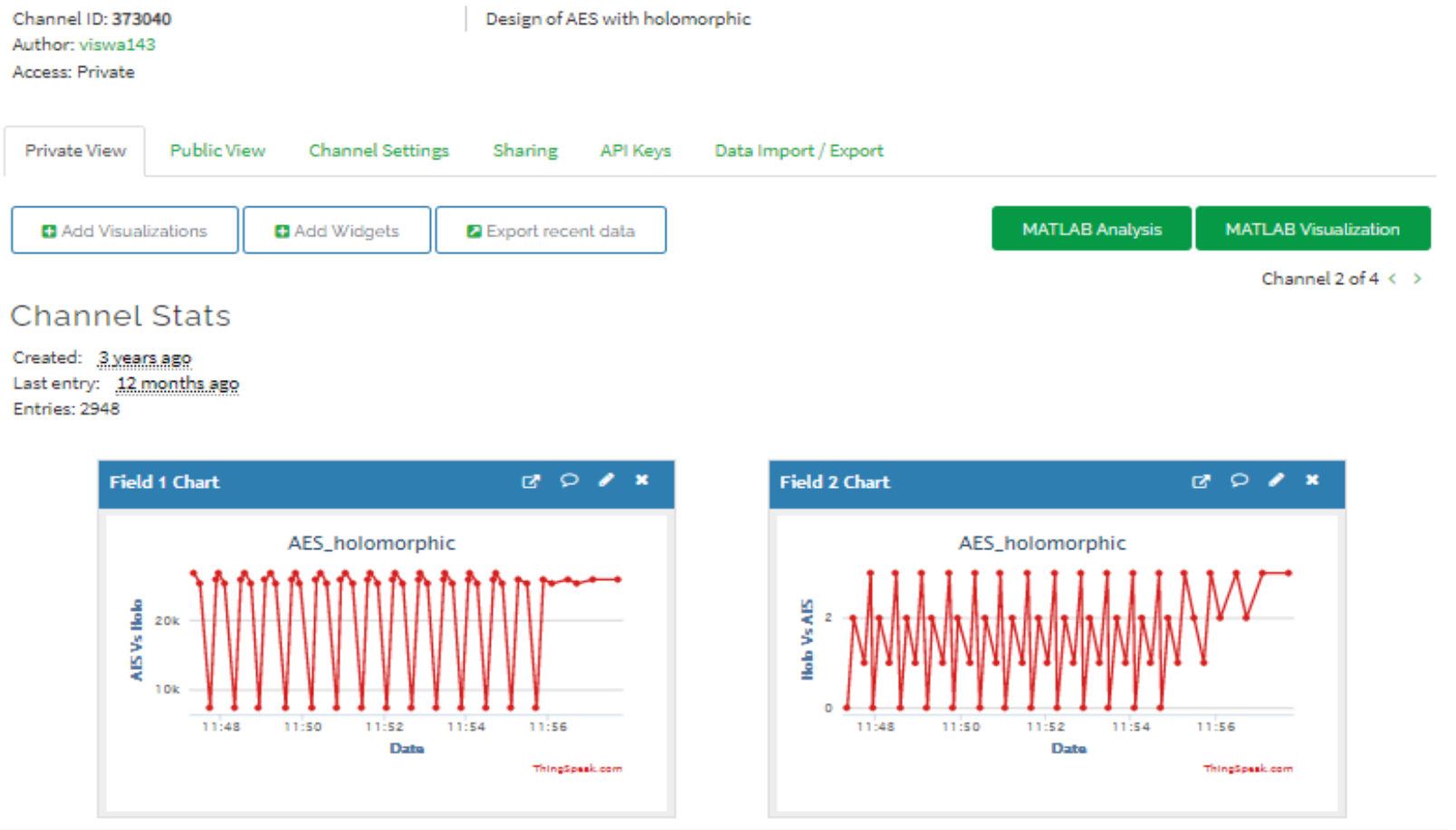

Fig.4. Simulated results of proposed hybrid techniques to secure the data, holomorphic operations

\begin{tabular}{|c|c|c|c|c|c|}
\hline Timestamps & $\begin{array}{l}\text { Variable: } 1 \\
\text { Data as Input }\end{array}$ & $\begin{array}{l}\text { Variable: } 2 \\
\text { No of Slots }\end{array}$ & \multirow{2}{*}{$\begin{array}{l}\text { Encrypted } \\
\text { Data in } \\
\text { binary } \\
\text { format }\end{array}$} & $\begin{array}{l}\text { Decoded } \\
\text { Data }\end{array}$ & $\begin{array}{l}\text { Throughput } \\
\text { in } \mathrm{GHz}\end{array}$ \\
\hline \multicolumn{2}{|c|}{ Conversion using Holomorphic } & & & $\begin{array}{l}\text { Decryption } \\
\text { Cloud }\end{array}$ & Process in \\
\hline 06-Apr-2020 18:54:05 & 27101 & 0 & $\begin{array}{llll}1 & 1 & 0 & 0 \\
1 & 0 & 1 & 0 \\
1 & 0 & 0 & 1 \\
0 & 1 & 1 & 1 \\
0 & 1 & 1 & 0 \\
\end{array}$ & 27101 & 0.2559 \\
\hline 06-Apr-2020 18:55:05 & 24121 & 1 & & 24121 & \\
\hline 06-Apr-2020 18:56:05 & 9825 & 2 & & 9825 & \\
\hline 06-Apr-2020 18:57:05 & 19969 & 3 & & $\begin{array}{l}29805 \\
\text { (Lose in data) }\end{array}$ & \\
\hline
\end{tabular}

Table.2. Comparison between proposed work and previously published work

\begin{tabular}{|l|l|l|}
\hline Parameter & Proposed & Existing \\
\hline Execution time & $184 \mathrm{~ms}$ & $230 \mathrm{~ms}[31]$ \\
\hline Throughput & $741 \mathrm{MHz}$ & $620 \mathrm{MHz}[33]$ \\
\hline Packet delivery rate & $402 \mathrm{MHz}$ & $372 \mathrm{MHz}[34]$ \\
\hline SNR & 86 & 82 \\
\hline PNSR & 93 & 89 \\
\hline MSE & 0.32 & 0.56 \\
\hline
\end{tabular}




\section{CONCLUSION}

The proposed new technique for cryptography is more feasible until protection, rapidity, power is complex as the structure employs only XOR and simple experimental functions which use few resources. The bit process for both enciphering and deciphering is reduced, in some of the applications such as Network operation Center and Security operation Center chips, power is used for the functioning of wired and distant communication used for rapid switching action among any twoinformation gathering and communication. ECCHE for deciphering and enciphering is primarily reduced, power consumption and ECCHE are combined with ECC which are used for exceptional secret keys to encipher and decipher the constant data via wired or distant channels. The recommended design is executed using MATLAB 2014a. To specify the limitations of the prior work a new system is designed for guaranteeing the security and protection of the information maintained in the cloud. This structure consists of three modules, access policy control, encryption, and decoding. Similarly, it comprises elements such as information manager (college administrator), CSP, and information client. Initially, the information user encrypts the information by using the ECCHE algorithm, and the cloud server store the information. Hence, the information client can provide access control to obtain the information maintained in the cloud. The proposed strategy authenticates the entrance control approach of the stated client for allowing limited access to the information. Currently, the private and public keys employed for information encryption are produced by selecting the 4-piece irregular whole numbers. During encryption, the quadratic key modules are considered and the arbitrary number is contemplated as the disturbance. Hence, the Holomorphic responsibilities are realized and expansions are performed on the integer information. As soon as the cited client obtains the information, the decoding computation is applied to transform the integer information into the original information.

\section{REFERENCES}

[1] Jian Xu.et.al, "SPCSS: Social Network-Based Privacy-Preserving Criminal Suspects Sensing", IEEE TRANSACTIONS ON COMPUTATIONAL SOCIAL SYSTEMS, DOI: 10.1109/TCSS.2019.2960857, 2329-924X, 2020 IEEE.

[2] Y. GovindaRamaiah, G. VijayaKumari, "Efficient public key generation for homomorphic encryption over the integers", $3^{\text {rd }}$ International Conference on Advances in Communication, Network and Computing, CNC-2012, Janahan Lal Stephen (Ed.), LNICST, pp. 262-268, Springer, 2012
[3] Mingyue Shi.et.al. "A privacy protection method for health care big data management based on risk access control", Health Care Management Science, https://doi.org/10.1007/s10729-019-09490-4, Springer, 2019.

[4] P. Scholl, N.P. Smart, "Improved key generation for Gentry's fully homomorphic encryption Scheme", Cryptology ePrint Archive: Report 2011/471, http://eprint.iacr.org/2011/471.

[5] C. Gentry, S. Halevi, "Fully homomorphic encryption without squashing using depth-3 arithmetic circuits", Cryptology ePrint Archive: Report 2011/279. http://eprint.iacr.org/2011/279.

[6] P. Scholl, N.P. Smart, "Improved key generation for Gentry's fully homomorphic encryption Scheme", Cryptology ePrint Archive: Report 2011/471, http://eprint.iacr.org/2011/471

[7] H. Jing-Li, Y. Ming, W. Zhao-Li, "Fully homomorphic encryption scheme extended to large message space", International Conference on Instrumentation, Measurement, Computer, Communication and Control, pp.533-536, IEEE, 2011.

[8] Shin-Yi Lin," A High-Throughput Low-Power AES Cipher for Network Applications",pp. 1-4244-06307/07/2007 IEEE.

[9] Shin-Yi Lin," A High-Throughput Low-Power AES Cipher for Network Applications",pp. 1-4244-0630.

[10] Y. GovindaRamaiah, G. VijayaKumari, "Efficient public key generation for homomorphic encryption over the integers", $3^{\text {rd }}$ International Conference on Advances in Communication, Network and Computing, CNC-2012, Janahan Lal Stephen (Ed.), LNICST, pp. 262-268, Springer, 2012.

[11] Arunkumar, Dr. S.S. Tyagi, ManishaRana, NehaAggarwal, PawanBhadana, ManavRachna A Comparative Study of Public Key Cryptosystem based on ECC and RSA, International Journal on Computer Science and Engineering (IJCSE), 2011 International University, Faridabad, India.

[12] Riaz Naseer and Jeff Draper, "Parallel Double Error Correcting Code Design to Mitigate Multi-Bit Upsets in SRAMs," 978-1-4244-2361-3/08/\$25.00 C2008 IEEE. Abbreviation

[13] Juan Antonio Maestro, Pedro Reviriego, SanghyeonBaeg, Shijie Wen, Richard Wong, "Soft error-tolerant Content Addressable Memories (CAMs) using error detection code and duplication," @2013 Elsevier B.V.

[14] SanghyeonBaeg, Pedro Reviriego, Juan Antonio Maestro, Shijie and Richard Wong, "Analysis of a Multiple Cell Upset Failure Model for Memories".

[15] Sandeep M D and Rajashekhargouda C. Patil, "An Approach to Reduce Number of Redundant Bits used To Overcome Cell Upsets in Memory using Decimal Matrix Code," Proc. Of Int. Conf. on Recent Trends in Signal Processing, Image Processing, and VLSI, ICrtSIV, ACEEE,2014. 
[16] Costas Argyrides, StephaniaLoizidou, and Dhiraj K. Pradhan, "Area Reliability Trade-Off in Improved Reed-Muller Coding," SAMOS 2008, LNCS 5114, pp. 116-125, 2008 Springer - Verlag Berlin Heidelberg 2008.

[17] Bertozzi, D., et al. "Error control schemes for on-chip communication links: the energy-reliability tradeoff". IEEE Trans. on DAC 24(6) (June 2005).

[18] Rossi, D., Metra, C. "Error-correcting strategy for high speed and density reliable flash memories". IEEE J. Electronic Testing, Theory and Applications 19(5), 511-521 (2003).

[19] F.Amounas and E.H.ElKinani, "Fast mapping method based on matrix approach for elliptic curve cryptography", International journal of information and network, Vol.1, No.2. Victor S. Miller, Use of elliptic curves in cryptography, In Proceeding of the Advances in Cryptology-Crypto'85, LNCS, SpringerVerlag, pp. 417-426, 1985.

[20] Do-Hyeon Choi, Hyungjoo Kim, 3Jungho Kang, 4Moonseog Jun, ECC-based Mobile WIMAX Initial Network Entry with Improved Security, International Journal of Advanced Computer Technology (IJACT): Vol. 5, No. 13, pp. $505 \sim 517,2019$

[21] Swadeep Singh, AnupriyaGarg, AnshulSachdeva, "Comparision of Cryptographic Algorithms ECC and RSA", International Journal of Computer Science and Communication Engineering (IJCSC), Special issue on "Recent Advances in Engineering \& Technology” NCRAET2013, ISSN 2319-708

[22] P. K. Shau, Dr. R. K. Chhotray, Dr. Gunamani Jena, Dr. S Pattnaik, "An Implementation of Elliptic Curve Cryptography”, International Journal of Engineering Research and Technology (IJERT) ISNN: 2278-0181, Vol 2 Issue 1, January 2013.

[23] M. Kurt, T. Yerlikaya, "A New Modified Cryptosystem Based on Menezes Vanstone Elliptic Curve Cryptography Algorithm that Uses Characters' Hexadecimal Values", TAEECE 2013, Konya, Turkey, 2013.

[24] K. Viswanath and R. Gunasundari, "Analysis and Implementation of Kidney Stone Detection by Reaction-Diffusion Level Set Segmentation using Xilinx System Generator on FPGA", International Journal of VLSI Design, Volume 2015, Article ID 581961, pp. 1-10. Hindawi Publishing Corporation, DOI: dx.doi.org/10.1155/2015/581961.
[25] Darel Hankerson and Alfred Menezes and Scott Vanstone, Guide to Elliptic Curve Cryptography, Springer-Verlag, 2004

[26] Fatima Amounas, El.Hassan El Kinani, and Chillali, "An application of discrete algorithms in asymmetric cryptography", International Mathematical Forum 6 (49), pp. 2409-2418, 2011.

[27] K Subba Reddy and V Uday Kumar “A Practical Approach for Secured Data Transmission using Wavelet based Steganography and Cryptography", International Journal of Computer Applications. ISSN (0975 - 8887) Volume 67- No.10, April 2013.

[28] M Purusotham Reddy "Host-Based Information Gathering Honeypots for Network Security", International Journal of Computational Engineering \& research. ISSN 2250-3005 Volume 2- Issue No.2, April 2012.

[29] T. Wollinger, J. Guajardo, and C. Paar, "Security on FPGAs: State-of the-art implementations and attacks," IEEE Trans.EmbeddedComput. Syst., vol. 3, no. 3, pp. 534-574, Aug. 2004.

[30] Do-Hyeon Choi, Hyungjoo Kim, 3Jungho Kang, 4Moonseog Jun, ECC-based Mobile WIMAX Initial Network Entry with Improved Security, International Journal of Advanced Computer Technology (IJACT): Vol. 5, No. 13, pp. $505 \sim 517,2019$.

[31] M. IndraSena Reddy and M. Subba Reddy, "Key Distillation process on Quantum cryptography protocols in Network Security", International Journal of Advanced Research computer science and Software Engineering, Vol.2, Issue 6, 2012

[32] K. Viswanath and R. Gunasundari, "VLSI implementation and analysis of Kidney Stone Detection of Ultrasound Image by Level Set Segmentation and MLPBP ANN Classification", Special issue on Artificial Intelligence and Evolutionary Computations in Engineering Systems, Advances in Intelligent Systems and Computing, pp. 208-227, Springer, India 2016, DOI 10.1007/978-81322-2656-7 19.

[33] K. Viswanath and R. Gunasundari, "Modified Distance Regularized Level Set Segmentation based analysis for kidney stone detection", International Journal of Rough Sets and Data Analysis, 2 (2), 2239, July-December 2015. DOI: 10.4018/ijrsda. 2015070102.

\section{Creative Commons Attribution License 4.0 (Attribution 4.0 International, CC BY 4.0)}

This article is published under the terms of the Creative Commons Attribution License 4.0 https://creativecommons.org/licenses/by/4.0/deed.en US 